\title{
Spectral responsivity of single-quantum-well photodetectors
}

\author{
S. A. Vitusevich, ${ }^{\text {a) }}$ A. Förster, W. Reetz, and H. Lüth \\ Institut für Schicht- und Ionentechnik, Forschungszentrum Jülich, D-52425 Jülich, Germany
}

A. E. Belyaev and S. V. Danylyuk
Institute of Semiconductor Physics, National Academy of Sciences of Ukraine, Kiev 252028, Ukraine

(Received 7 February 2000; accepted for publication 9 May 2000)

\begin{abstract}
The photoresponsivity spectra of double-barrier resonant tunneling diodes have been measured in a wide range of light wavelength as well as applied voltage. The complex behavior of measured spectra is analyzed taking into account different channels for electron injection into the quantum well (QW). It has been shown that the photoresponse in the infrared wavelength range could arise not only from the electrons excited inside the QW, but also from the two-dimensional electrons confined on the quantum level in the spacer accumulation layer. (c) 2000 American Institute of Physics. [S0003-6951(00)03127-2]
\end{abstract}

Over the last decade resonant tunneling diodes based on double-barrier heterostructures attract an elevated attention due to their electronic and photonic applications. The latter were concentrated on utilization of the unique electronic and optic properties of these devices for lasers, detectors, and modulators in the infrared to the visible wavelength range, ${ }^{1,2}$ and high-speed optically switched electronic devices. ${ }^{3,4}$ At the same time investigation of such devices under illumination could be successfully used for the understanding and analysis of basic electronic processes occurring in vertical transport devices.

This letter presents photoresponsivity measurements done as a function of excitation wavelength on a GaAs/AlAs double-barrier resonant tunneling diode. The results obtained at various bias voltages help to elucidate the relative contributions to the tunnel current from carriers directly injected into the well from three-dimensional (3D) emitter, versus those that tunnel in from an accumulation layer formed in front of the first barrier. Futhermore, they yield evidence for modulated electron-hole (e-h) recombination in the well provided by direct excitation of e-h pairs in the well.

The resonant tunneling diodes (RTDs) we have studied were manufactured with the use of a GaAs/AlAs doublebarrier heterostructure grown by molecular beam epitaxy on $n^{+}-(100) \mathrm{GaAs}$ substrate. The active part of the RTD studied in this work consists of a 4-nm-thick GaAs quantum well (QW) with 2-nm-thick AlAs barriers on both sides. These three layers were nominally undoped. A 100-nm-thick undoped GaAs spacer layer was grown adjacent to the barrier on the substrate side and was separated from the substrate by a 100 -nm-thick highly doped $\left(\sim 10^{18} \mathrm{~cm}^{-3} \mathrm{Si}\right) n^{+}$-GaAs electrode region. A 600-nm-thick GaAs heavy doped top layer of similar doping was grown directly on top of the other barrier. The $16 \times 16 \mu \mathrm{m}^{2}$ square devices were defined by wet-chemical mesa etching and $\mathrm{Au} / \mathrm{Ge} / \mathrm{Ni}$ alloyed ohmic contacts were fabricated on the substrate and on top of the mesa to provide measurements under applied bias.

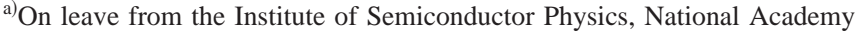
of Sciences of Ukraine, Kiev 252028, Ukraine; electronic mail: s.vitusevich@fz-juelich.de
}

The responsivity spectra of the RTD were measured using a standard spectral response system based on a $450 \mathrm{~W}$ xenon lamp and a grating monochromator. The bandwidth of the monochromatic light is $20 \mathrm{~nm}$ and the wavelength is changed in the 400-900 nm wavelength range in $10 \mathrm{~nm}$ steps. A calibrated single crystalline silicon detector is used for the system calibration which gives the light intensity at each wavelength position. The RTD was illuminated by light incident at $45^{\circ}$ on the sidewall facet. With an incident optical power of $30 \mathrm{nW}$, corresponding to an irradiance of $0.043 \mathrm{~mW} / \mathrm{cm}^{2}$ we did not observe visible changes in current-voltage $(I-V)$ characteristics. Thus, the optically generated carriers did not significantly modify the potential distribution within the device.

Typical responsivity spectra of the RTD, measured at 77 $\mathrm{K}$ and with different applied bias, are shown in Fig. 1. These spectra reveal a narrow band (labeled $A$ in Fig. 1) at $1.5 \mathrm{eV}$ and a broadband (labeled $B$ in Fig. 1) at $1.65 \mathrm{eV}$ with an extended short-wavelength tail. The intensities of both bands strongly depend on applied voltage and qualitatively follow the dark current dependence of the $I-V$ curve (Fig. 2). The $I-V$ characteristics of the RTD measured in the dark reveals three resonant peaks under forward bias (Fig. 2), instead of the two peaks which actually appeared at reversed bias polarity. In all measurements the forward bias corresponds to a

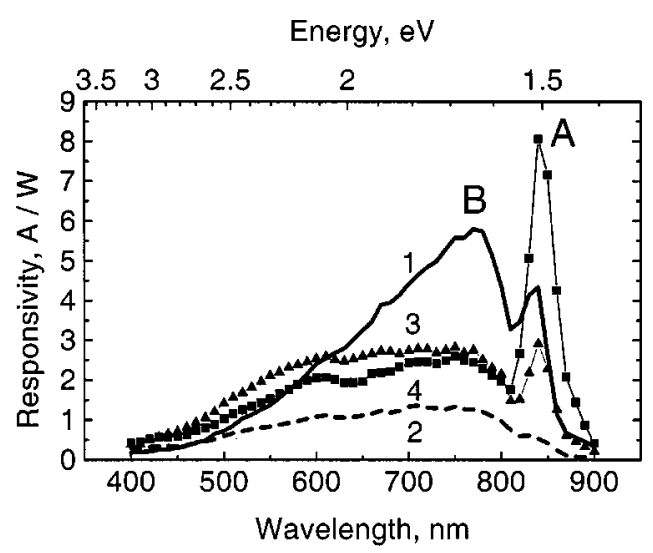

FIG. 1. Photoresponsivity spectra measured at $T=77 \mathrm{~K}$ on the RTD biased at: 1-0.6, 2-0.8, 3-1.7, and 4-2.0 V. 


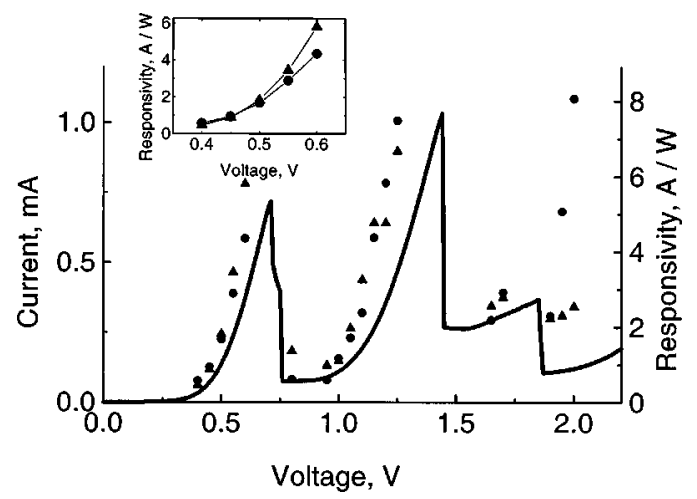

FIG. 2. $I-V$ characteristics of the RTD measured at $T=77 \mathrm{~K}$ under forward bias. Solid circles and triangles show the intensity of the photoresponse corresponding to maxima of the bands $A$ (circles) and $B$ (triangles). Enlarged dependence of these values within the first resonance is shown on inset.

negatively biased substrate. Previously we suggested that the first two peaks observed for both bias polarities arise as a result of tunneling of electrons injected from the 3D emitter through the first and second quasibound states of the QW, while the third peak observed at forward bias is due to electrons injected from a two-dimensional (2D) level in the accumulation layer formed in front of the emitter barrier. ${ }^{5}$ Further we will concentrate on results obtained under forward bias.

In the band-to-band excitation case with energy close to GaAs band gap the e-h pairs are creating throughout the structure outside the QW. The e-h pairs generated in the collector are shifted by the electric field. Electrons move towards the doped layer and recombine, thus do not contribute to the photocurrent, while the holes move to the collector barrier and are accumulated there. These carriers could tunnel throughout the double barrier structure directly or overcome the barrier thermionically. The latter could be neglected due to the height of the barriers and the low temperature. The tunneling holes recombine with electrons on the emitter side and thus contribute to the photocurrent. In contrast, the electrons photogenerated on the emitter side are injected into the QW. The magnitude of photoresponse increases with applied voltage under resonant conditions while it is low at biases corresponding to the resonance-off conditions. On the spectral dependence of the photoresponse the processes mentioned earlier should reveal themselves as a narrow asymmetric band with cutoff corresponding to the GaAs gap energy. That is because with increasing energy of the incident photons we should expect a sharp decrease of the photocurrent due to a high absorption coefficient and, consequently, of a small amount of photogenerated pairs. Surprisingly, a wide band has been observed on the shortwavelength side, as it is seen in Fig. 1. The shape of the band is strongly affected on the applied voltage. There is a pronounced maximum at $1.65 \mathrm{eV}$ followed by a shortwavelength tail within the first resonance, while the band becomes more flat with the center of gravity shifted towards higher excitation energy within the second resonance and, finally, it almost vanishes at voltages corresponding to a carrier transfer above the barrier. The behavior of the spectral response like that could be explained with the following arguments. If the energy of the incident photons coinsides with Downloaded 15 Dec 2006 to 134.94.122.39. Redistribution subject the energy difference between electronic and hole quasibound states in the QW, a direct generation of e-h pairs inside the QW is possible. The photocarriers are removed out of the QW by the electric field remaining behind "holes" which are filling immediately by electrically injected electrons from the emitter as well as holes from the accumulation layer adjacent to the collector barrier thus giving rise to a photocurrent. Such a process should be efficient at resonance, while it is insignificant in the offresonance regime because of a low probability to supply carriers into the QW. This implies a strong field dependence of the photocurrent.

It is worth to note that there is an essential limitation of the photocurrent intensity related to the recombination of the photogenerated carriers. It has been reported earlier ${ }^{6}$ that a QW photoluminescence (PL) in double-barrier structures reveals field-dependent behavior. Moreover, Vodjdani et al. ${ }^{7}$ have shown that the radiative recombination does occur in the QW and roughly follows the current variation with bias either the e-h pairs electrically injected or photocreated directly in the QW. At the same time the PL dynamics directly reflect the source for holes participating in the recombination. The study of time-resolved PL from excitons in the QW shows that the net holes lifetime inside the QW is much shorter if the holes are created directly in the QW. ${ }^{8}$

Now, it becomes clear why the peak intensity of the band $B$ (Fig. 1) grows more quickly that one of the band $A$ under voltage region corresponding to the first resonance. Indeed, the QW luminescence is sublinear in the electron density and saturates at high electron injection. Taking into account the difference between the lifetime for holes generated by different processes, we can conclude that a saturation occurs at lower bias in the case of the e-h pairs generation directly in the QW. The fact is confirmed by the $R_{p}{ }^{A, B}$ $=f(V)$ dependences plotted in the inset of Fig. 2. On the other hand, in the voltage region corresponding to the second resonance the recombination will be determined by the number of electrons which came out from the resonance and relaxed onto the ground quasibound state of the QW. This amount, obviously, is smaller than is necessary for a saturation and the $R_{p}{ }^{A, B}=f(V)$ dependences almost coincide.

It should be noted that the spectral responsivity data obtained with illumination of the RTD in the wavelength range corresponding to interband transitions do not allow to distinguish directly either carriers injected into the $\mathrm{QW}$ from $3 \mathrm{D}$ or 2D channels. Nevertheless, an explanation of the experimental results given earlier is more consistent with ballistic transport of tunneling electrons. An additional evidence that proves our conclusion is the observation of fine oscillatory structure of the tunnel current flowing through the RTD under resonance conditions. The oscillations are still observable and their period does not change with illumination as it is seen from Fig. 3. A number of mechanisms have been proposed to explain the fine structure of the tunnel current. In spite of their differences, most of mechanisms proposed associate periodicity with electron scattering on longitudinal optical phonons, whose energy is $36 \mathrm{meV}$. This assumption is not appropriate in our case because the period of oscillations for our structure is definitely less than $36 \mathrm{meV}$. Taking into account the scaling factor that converts the applied voltage into the energy shift we can estimate the energy differto AlP license or copyright, see http://apl.aip.org/apl/copyright.jsp 


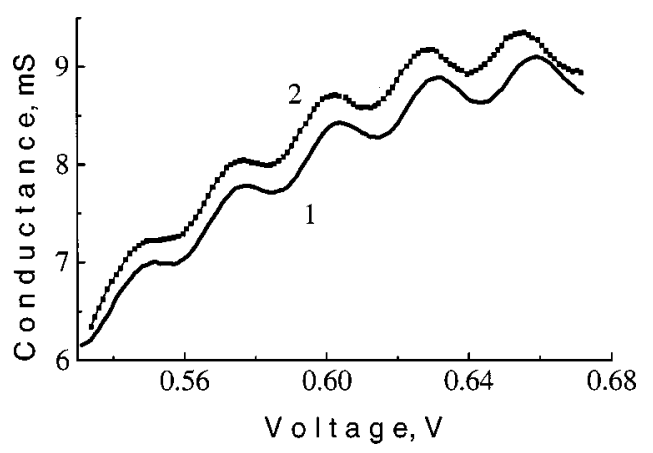

FIG. 3. Conductance vs applied voltage dependence measured in the dark (curve 1) and under illumination (curve 2).

ence between neighboring maxima as $6 \mathrm{meV}$. To explain the observed behavior we suggested a model based on quantum interference effects arising in structures having a wide spacer layer. ${ }^{5}$ The model predicts the appearance of a fine structure in the tunnel current and the period of oscillations is determined exclusively by the structure design. In terms of wave mechanics, we have an additional resonator (the spacer region) in front of the Fabry-Perot interferometer (the doublebarrier structure). As a result, the transmission coefficient of resonant tunneling through the $\mathrm{QW}$ is modulated by interference in the spacer if transport in it is ballistic.

An accumulation layer plays a more important role at high voltages and can be checked by photocurrent measurements. Indeed, the power of excitation used does not disturb a potential distribution along the device within the first and second resonances, while it stimulates visible changes of the $I-V$ characteristics at bias exceeding $1.5 \mathrm{~V}$ leading to a shift of the third peak position and to a variation of its magnitude. The character of the changes depends on the excitation energy. In most cases the excitation with photons whose energy corresponds to interband transitions caused a shift of the resonance towards higher voltage and, simultaneously, an increase of the current. In contrast, intersubband excitation led to opposite changes. In Fig. 4 we plotted the resposivity of the RTD measured in the wavelength range from 1.3 to $1.9 \mu \mathrm{m}$. The spectral shape of the responsivity spectrum is essentially changed for different biases. The curve measured at $0.7 \mathrm{~V}$ shows an intensive band at $1.73 \mu \mathrm{m}$. A shoulder on the short-wavelength side is also visible. At $2.25 \mathrm{~V}$ the curve is transformed in a two-band spectrum with an intensive band at $1.67 \mu \mathrm{m}$ and a weaker one at $1.83 \mu \mathrm{m}$. All these features we describe as photoinduced bound-to-continuum transitions. At low bias they correspond to excitation of electrons from localized state in the accumulation layer (shoulder, transition 1 in inset of Fig. 4) and ground quasibound state in the QW (band, transition 2 in inset of Fig. 4). In both cases the height of the barriers that could be overcame by the excited carriers is in good agreement with the potential profile calculated for the given structure. Indeed, we have two subbands of the QW with minima at 0.18 and $0.68 \mathrm{eV}$. Conduction-band offset for GaAs/AlAs heterostructure

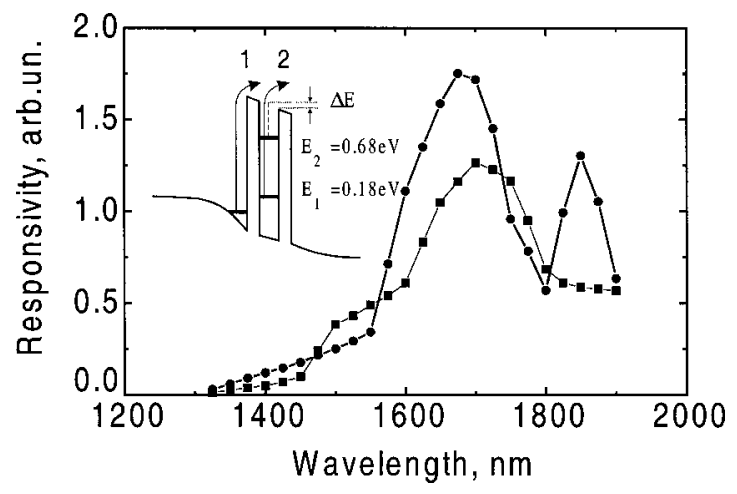

FIG. 4. Spectra of the photoresponse measured at $T=77 \mathrm{~K}$ in the wavelength range from 1300 to $1900 \mathrm{~nm}$ at voltages: $0.7 \mathrm{~V}$-squares, $2.25 \mathrm{~V}$-circles. Inset shows potential profile of the structure and origin of excited carriers marked by arrows.

brings the barrier height of $1 \mathrm{eV}$. The electric-field-induced barrier lowering, $\Delta E=e F w / 2$, where $F$ is the electric field and $w$ is the well width, could be of order $0.1 \mathrm{eV}$ in electric fields of $10^{-7}-10^{-8} \mathrm{~V} / \mathrm{m}$ that are realistic values. Thus, the bound-to-continuum transition should be expected at 1.73 $\mu \mathrm{m}$. At high bias the energy needed for electrons to be escaped decreases due to lowering of the effective height of the barriers with the increase of the electric field. Moreover, the efficiency for electron excitation from the localized state in the accumulation layer grows considerably and the shoulder in the responsivity spectra transforms into a well pronounced band.

In conclusion, we have observed considerable transformation of the responsivity spectra in double-barrier RTD resulting on different origin of the photogenerated carriers. Besides, it has been shown that the photoresponse in the infrared wavelength range could arise not only from the electrons excited inside the $\mathrm{QW}$, but from the ones localized in the accumulation layer also. The fact should be taken into account when QW infrared photodetectors are designed.

One of the authors (S.A.V.) would like to acknowledge the support by the Alexander von Humboldt Foundation. A.E. Belyaev acknowledges DAAD for Research Fellowship.

${ }^{1}$ B. F. Levine, J. Appl. Phys. 74, R1 (1993).

${ }^{2}$ A. Rogalski, Infrared Phys. Technol. 38, 295 (1997).

${ }^{3}$ H. S. Li, L. P. Chen, Y. W. Chen, K. L. Wang, D. S. Pan, and J. M. Liu, Appl. Phys. Lett. 65, 2999 (1994).

${ }^{4}$ T. S. Moise, Y.-C. Kao, L. D. Garrett, and J. C. Campbell, Appl. Phys. Lett. 66, 1104 (1995).

${ }^{5}$ A. E. Belyaev, S. A. Vitusevich, T. Figielski, B. A. Glavin, R. V. Konakova, L. N. Kravchenko, A. Makosa, and T. Wosinski, Surf. Sci. 361/362, 235 (1996).

${ }^{6}$ M. S. Skolnick, P. E. Simmonds, D. G. Hayes, A. W. Higgs, G. W. Smith, A. D. Pitt, C. R. Whitehouse, H. J. Hutchinson, C. R. White, L. Eaves, M. Henini, and O. H. Hughes, Phys. Rev. B 42, 3069 (1990).

${ }^{7}$ N. Vodjdani, D. Cote, D. Thomas, B. Sermage, P. Bois, E. Costard, and J. Nagle, Appl. Phys. Lett. 56, 33 (1990).

${ }^{8}$ S. Charbonneau, J. F. Young, and A. J. SpringThorpe, Appl. Phys. Lett. 57, 264 (1990). 